\title{
Anaerobic fermentation of glycerol: a platform for renewable fuels and chemicals
}

\author{
James M. Clomburg' and Ramon Gonzalez ${ }^{1,2}$ \\ ${ }^{1}$ Department of Chemical and Biomolecular Engineering, Rice University, 6100 Main Street, MS-362, Houston, TX 77005, USA \\ ${ }^{2}$ Department of Bioengineering, Rice University, Houston, TX, USA
}

\begin{abstract}
To ensure the long-term viability of biorefineries, it is essential to go beyond the carbohydrate-based platform and develop complementing technologies capable of producing fuels and chemicals from a wide array of available materials. Glycerol, a readily available and inexpensive compound, is generated during biodiesel, oleochemical, and bioethanol production processes, making its conversion into value-added products of great interest. The high degree of reduction of carbon atoms in glycerol confers the ability to produce fuels and reduced chemicals at higher yields when compared to the use of carbohydrates. This review focuses on current engineering efforts as well as the challenges involved in the utilization of glycerol as a carbon source for the production of fuels and chemicals.
\end{abstract}

\section{The potential of glycerol}

The search for new technologies able to generate renewable fuels and chemicals has gained significant momentum in recent years, due in large part to the rising concerns related to the cost, sustained availability, and environmental impact of fossil fuels [1,2]. The availability of diverse biomass resources, such as agricultural lignocellulosic residues and edible and non-edible crops, has resulted in significant advancements in the conversion of biomass into renewable fuels and chemicals through the implementation of different technologies capable of utilizing specific biomass feedstock constituents [3-8]. Although the production of biofuels such as bioethanol from the most commonly used feedstocks on an industrial scale (starches and simple sugars derived from sources such as sugar cane and corn) is highly efficient [5], these feedstocks are expensive and non-sustainable due to their concurrent integration as an essential component of the food-feed chain. Even lignocellulosic crops, although highly productive, sustainable, and renewable, are difficult to convert into sugars leading to higher feedstock and operating costs [4,5]. In addition, similar economic and political factors have led to an increase in the feedstock and operating costs for biodiesel production that, at times, result in feedstock and operating costs that nearly match the market price of biodiesel [9].
To increase the economic viability for the future production of biofuels, the concept of a biorefinery has been proposed (Figure 1) [10]. The concept of a biorefinery is analogous to the way a petroleum refinery produces multiple fuels and products from a single feedstock. However, unlike current petroleum refineries, biomass would be used as the main feedstock. By using both byproduct streams and a small portion of the feedstock to produce higher value, small market chemicals along with the biofuels, the value derived from the biomass feedstock can be maximized.

One promising avenue for the coupling of processes within a biorefinery is the utilization of glycerol as a substrate for the production of biochemicals and biofuels. Glycerol is an inevitable byproduct generated during both bioethanol and biodiesel production processes, and the tremendous growth of these industries has led to a dramatic decrease in crude glycerol prices over the past few years $[9,11,12]$ (Figure 2). During biodiesel production, the transesterification of fats and oils with an alcohol results in $10 \mathrm{lbs}$ of crude glycerol for every $100 \mathrm{lbs}$ of biodiesel produced. Furthermore, regardless of the feedstock used (i.e., sugar cane or corn), bioethanol produced through the fermentation of sugars by yeasts is accompanied with the generation of significant amounts of glycerol as a fermentation byproduct of these microorganisms. During a typical industrial bioethanol process, the ethanol is separated via distillation, and the remaining material (often called the column bottoms, vinasse, or whole stillage, depending on the feedstock) may be further processed to recover value from the byproducts [12]. The liquid fraction of the whole stillage (i.e., thin stillage) contains a complex mixture of chemicals, including up to $2 \%$ glycerol [12]. In addition to the large amounts produced by the bioethanol industry $[13,14]$, waste streams containing high levels of glycerol are generated in almost every industry that uses animal fats and vegetable oils as the starting material. For example, waste streams of 55-90\% glycerol are readily generated by the oleochemical industry and this additional surplus may further drop glycerol prices [9]. Up to $7.8 \mathrm{M}$ glycerol (equivalent to $718 \mathrm{~g} / 1$ glycerol in water) is also available in certain algae such as the Dunaliella genus [15]. The abundance of glycerol points to the need and potential for efficient, cost effective processes that convert glycerol into higher value fuels and chemicals. 


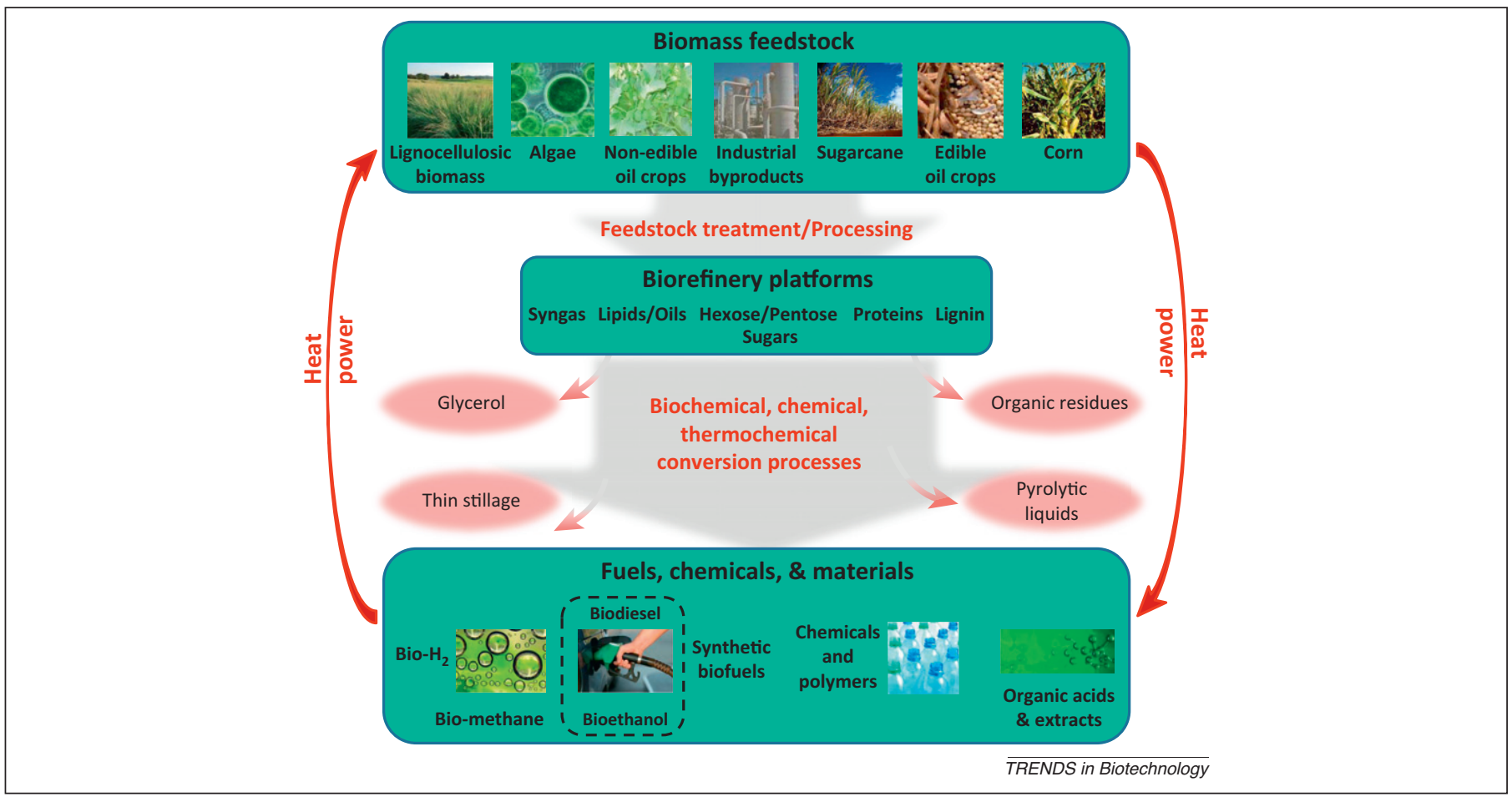

Figure 1. Generalized schematic of material and process integration within a biorefinery. Biomass is converted to platform intermediates and materials through various feedstock treatment and process techniques. Biochemical, chemical, and thermochemical processes such as fermentation, esterification, or gasification can then be utilized to synthesize fuels, chemical, materials, heat, and power from these intermediates. Byproducts of these processes (represented in red shading) can be re-integrated into the biorefinery as feedstocks, used for heat and power resources, or used directly as product (e.g., animal feed from organic residues). The current most widely produced

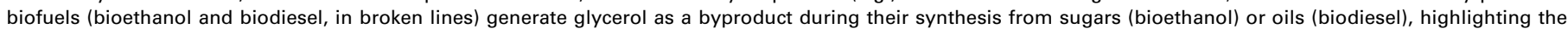
need and opportunity to convert glycerol to value-added fuels and chemicals within the integrated biorefinery framework.

The high degree of reduction of carbon atoms in glycerol $(\kappa=4.67$, where $\kappa$ represents the degree of reduction per carbon, a measure of the number of available electrons per unit of carbon [16]) provides a distinct advantage over more oxidized carbohydrate-based feedstocks such as glucose ( $=4)$ and xylose $(\kappa=4)$ when one considers the production of fuels and reduced chemicals. However, highly reduced glycerol is difficult for microorganisms to utilize under fermentative conditions (i.e., absence of external electron acceptors) (Box 1), a metabolic mode essential to fully

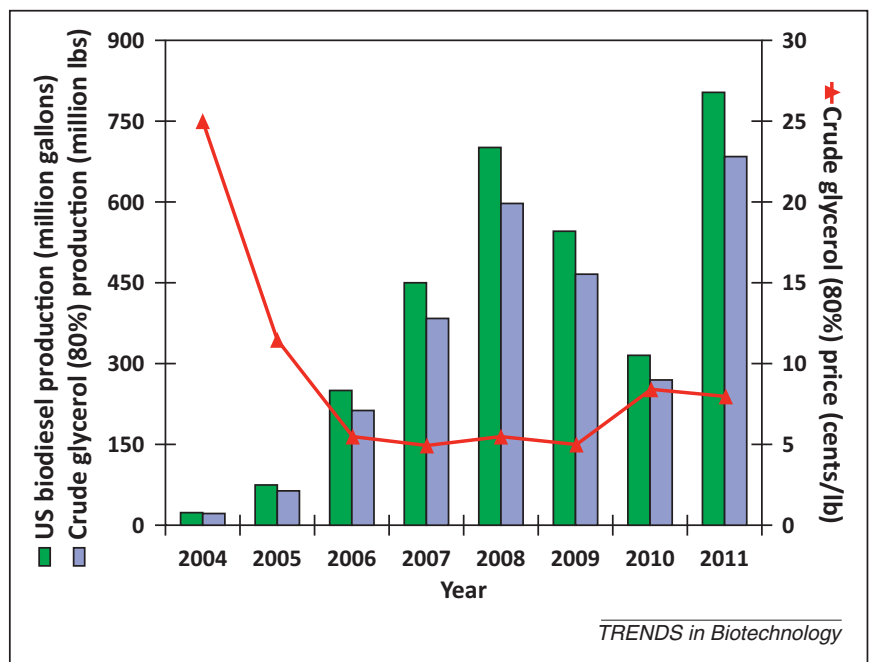

Figure 2. US biodiesel production and crude glycerol price (www. thejacobsen.com). exploit the reduced nature of glycerol. Here, we review recent engineering efforts designed to harness the potential of glycerol as a carbon source for the production of fuels and chemicals.

\section{Glycerol as a carbon source for the production of fuels and reduced chemicals}

During anaerobic fermentation, the overall redox balance within the cell is a key determinant dictating the overall yields and productivities that can be achieved during the synthesis of a specific product from a given carbon source. The conversion of glycerol into the metabolic intermediate pyruvate generates twice the number of reducing equivalents produced during the metabolism of lignocellulosic sugars such as glucose or xylose (Figure 3). These additional reducing equivalents provide glycerol with the natural advantage of higher theoretical product yields for reduced chemicals and fuels. For example, the use of glycerol permits the co-production of ethanol and formic acid (or ethanol and hydrogen), whereas the fermentation of sugars such as glucose dictates that about half of their weight is lost as $\mathrm{CO}_{2}$ reducing the product yield (Figure 3 ). Under fermentative conditions, redox poise must be maintained through the terminal transfer of electrons to internally generated organic compounds [17] and, as such, the natural shift toward reduced products during anaerobic fermentation of glycerol provides a means of maximizing the production of reduced chemicals and fuels [18]. Anaerobic fermentation also provides lower operating and capital costs than aerobic fermentation; anaerobic fermenters 
Box 1. Microbial utilization of glycerol under fermentative conditions

Although the highly reduced nature of carbon atoms in glycerol conveys many advantages for the production of fuels and reduced chemicals via microbial fermentation, these advantages also come with considerable challenges. The utilization of glycerol under fermentative conditions requires microorganisms that can metabolize such a reduced carbon source in the absence of external electron acceptors. Unlike traditional carbon sources such as glucose $(\kappa=4)$ and xylose $(\kappa=4)$, the degree of reduction per carbon, $\kappa$ [16], a measure of the number of available electrons per unit of carbon, of glycerol $(\kappa=4.67)$ is greater than that of cell mass ( $\kappa=4.3$, based on average biomass molecular formula of $\mathrm{CH}_{1.9} \mathrm{O}_{0.5} \mathrm{~N}_{0.2}$ [16]), illustrating that the formation of cell mass from glycerol will result in the generation of reducing equivalents (i.e., electrons). The ability of a microorganism to maintain overall redox poise, and hence possess the capability of utilizing glycerol as a carbon source under fermentative conditions, is directly tied to the ability to produce a product more reduced than glycerol, whose formation serves as a sink for the excess reducing equivalents generated during the formation of cell mass (Figure I). Therefore, whereas numerous microorganisms are able to utilize glycerol under respiratory conditions, few are capable of utilizing glycerol in the absence of external electron acceptors.

Fermentative metabolism of glycerol has been reported for several species of the Enterobacteriaceae family, including Escherichia coli, Citrobacter freundii, and Klebsiella pneumoniae, as well as species from the genera Clostridium, Lactobacillus, Bacillus, Propionibacterium, and Anaerobiospirillum [9].

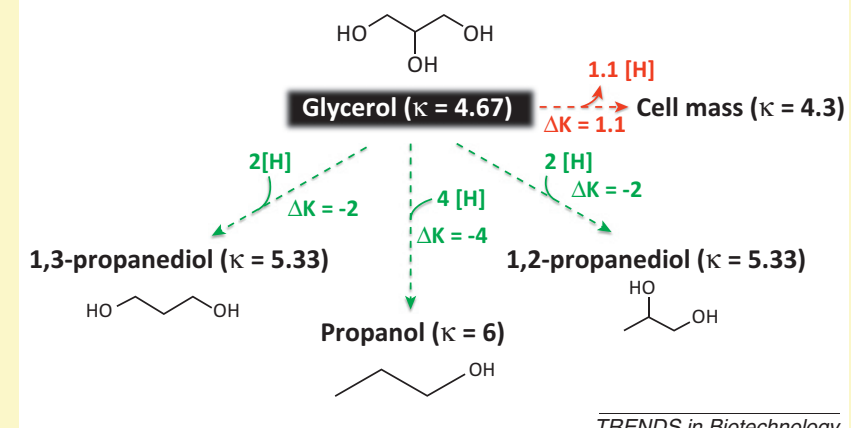

Figure I. Fermentative utilization of glycerol by microorganisms. The reducing equivalents generated during the formation of cell mass from glycerol (red line) must be consumed by the formation of a metabolic product more reduced than glycerol (green lines). The $\Delta \mathrm{K}$ value represents the degree of reduction balance of a given conversion. Abbreviation: $2[\mathrm{H}]=\mathrm{NADH} / \mathrm{NADPH} / \mathrm{FADH}{ }_{2}$.

are less expensive to build and operate and use less energy [9].

The majority of studies into fermentative production of fuels and chemicals from glycerol have focused on products naturally produced by organisms able to anaerobically ferment glycerol (Box 2). However, the potential for some of these microorganisms to produce at the industrial level could be limited due to pathogenicity, the requirement for strict anaerobic conditions, and the need of supplementation with rich nutrients. In addition, the lack of genetic tools and physiological knowledge necessary for the effective manipulation of certain organisms limits the ability to design effective metabolic engineering and synthetic biology strategies to improve the production of native products and expand the scope of available products to non-native compounds in these organisms. Consequently, the use of hosts more amendable to industrial applications is highly desirable. For example, the recent discovery that

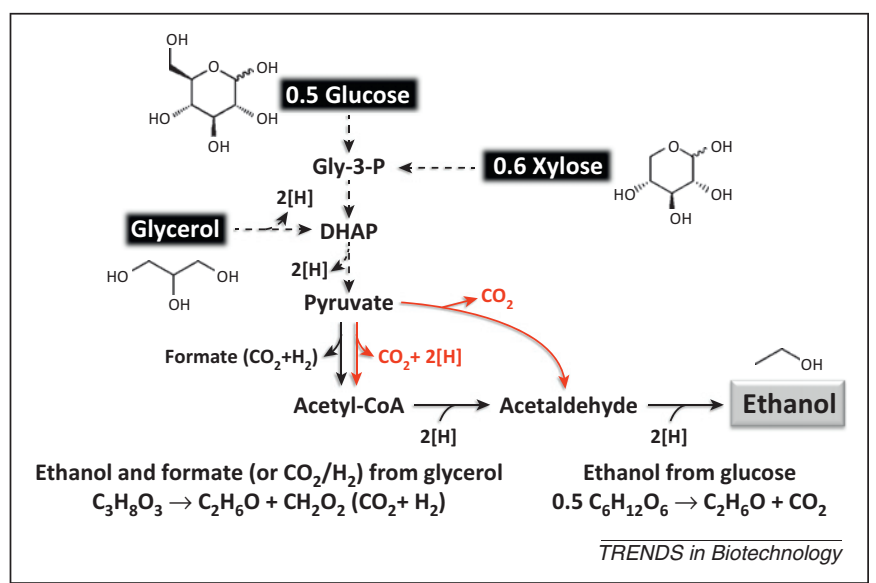

Figure 3. Ethanol production from glycerol, glucose, and xylose. The additional reducing equivalents generated from the conversion of glycerol into pyruvate permit the co-production of ethanol and formate (or $\mathrm{H}_{2}$ and $\mathrm{CO}_{2}$ ). The oxidation state of glucose and xylose dictates the conversion of pyruvate into ethanol must take place through the pathways indicated in red, leading to the loss of carbon as $\mathrm{CO}_{2}$. Broken lines represent multiple reaction steps. Abbreviations: DHAP, dihydroxyacetone-phosphate; Gly-3-P, glyceraldehyde 3-phosphate.

Escherichia coli is able to utilize glycerol in a fermentative manner [18,19] holds great promise for the industrial scale conversion of glycerol into a wide variety of value-added fuels and reduced chemicals. Below, we detail the current portfolio of fuels and chemicals produced during the fermentative of glycerol by native organisms as well as the metabolic engineering efforts to improve the production of target compounds.

\section{1,3-Propanediol (1,3-PDO)}

Fermentative utilization of glycerol was long thought to require the ability to produce 1,3-PDO (Box 2). Consequently, this diol molecule is the most extensively studied product of fermentative glycerol utilization. 1,3-PDO is used in solvents, adhesives, detergents, resins, and cosmetics. Over the past few decades it has gained attention as a monomer for the synthesis of the polyester polytrimethylene terephthalate (PTT), which has significant application in fibers, textiles, and carpets [20].

A number of microorganisms have been shown to ferment glycerol in a 1,3-PDO-dependent manner, with the biological production of 1,3-PDO from glycerol demonstrated in Lactobacillus brevis, Lactobacillus buchnerii [21,22], Bacillus welchii [23], Citrobacter freundii, Klebsiella pneumoniae [24,25], Clostridium pasteurianum [26], and Clostridium butyricum [27-29]. 1,3-PDO is more reduced than glycerol resulting in glycerol fermentation producing 1,3PDO and a more oxidized co-product. In Enterobacteriaceae, such as $C$. freundii and $K$. pneumoniae, glycerol fermentation results in the production of 1,3-PDO and acetic acid as the main fermentation products [24], whereas clostridial strains produce $1,3-\mathrm{PDO}$ along with a variety of other fermentation products including butyric acid, nbutanol, acetic acid, ethanol, and lactate depending on the strain and conditions [26-31].

The fermentative conversion of glycerol to 1,3-PDO with both natural producers and engineered strains has been very successful and a number of studies report high yields and titers of 1,3-PDO (Table 1). Anaerobic fermentation 
Box 2. Metabolic models for the fermentative utilization of glycerol in microorganisms

Metabolic models for the fermentative metabolism of glycerol encompass the genes, enzymes, and metabolic pathways that are directly linked to the ability of the microorganism to utilize glycerol as a carbon source. The most well characterized of these is the 1,3-PDO-dependent model in which the ability to utilize glycerol is directly linked to the synthesis of highly reduced 1,3-PDO ( $\kappa=5.33$ ) (Figure la). Fermentative metabolism of glycerol in several species of the Enterobacteriaceae family has been extensively studied and linked to the 1,3-PDOdependent model [78]. In these organisms, two parallel pathways are responsible for the dissimilation of glycerol into glycolytic intermediates and the synthesis of 1,3-PDO (Figure la) [79,80]. An oxidative pathway dehydrogenates glycerol to DHA through the action of a NAD-linked glycerol dehydrogenase, which is then phosphorylated by a PEP- or ATP-dependent DHA kinase. The phosphorylated product, DHA-phosphate, can then enter glycolysis, enabling the formation of essential metabolic intermediates and major fermentation products. A parallel reductive pathway converts glycerol to 1,3-PDO through the action of a coenzyme B12-dependent glycerol dehydratase and NADH-linked 1,3PDO dehydrogenase [80]. This reductive branch consumes NADH released by the formation of cell mass, thus providing a means for the cell to achieve a redox balance in the absence of external electron acceptors. The absence of 1,3-PDO-producing capability was previously thought to confine microorganisms to the use of glycerol only in the presence of external electron acceptors $[78,79]$. However, recent studies have reported fermentative glycerol utilization in Escherichia coli [66] and Paenibacillus macerans [61], microorganisms for which no 1,3-PDO production capability has been found. In these organisms, the ability to utilize glycerol under fermentative conditions is linked to the synthesis of 1,2-propanediol and ethanol as a means of facilitating redox balance and ATP generation, respectively (Figure Ib) $[19,61]$. This newly characterized model consists of an oxidative, type II glycerol dehydrogenase (glyDH-II) and PEP- or ATP-dependent DHAK-mediated pathway working in partnership with a reductive 1,2-PDO-producing pathway. The oxidative branch results in the conversion of glycerol into the glycolytic intermediate DHAP, a key node for both the reductive branch and ethanol synthesis.

In addition, other microorganisms have been shown to possess the ability to ferment glycerol with no reported propanediol-producing capability, including Propionibacterium acidipropionici [53] and Anaerobiospirillum succiniproducens [48]. However, the mechanisms and pathways mediating the dissimilation of glycerol under anaerobic conditions have not been investigated.

with glycerol as the sole carbon source has resulted in 1,3PDO titers greater than $60 \mathrm{~g} / \mathrm{l}$ from both isolated and engineered strains of $K$. pneumoniae [31-33], C. butyricum [31,34-37], and recombinant Clostridium acetobutylicum [34,38]. Due in part to the large number of studies and interest in the conversion of glycerol into 1,3-PDO, this topic has also been extensively reviewed [20,39]. Recently, a new screening method was developed to isolate bacteria exhibiting tolerance to high concentrations of both crude glycerol and 1,3-PDO [40]. One promising isolate, $C$. butyricum AKR102a, was utilized for the high level conversion of glycerol to 1,3-PDO during fed-batch fermentation with both refined and crude glycerol in the 1-liter and 200-liter scale [41]. Although capable of producing greater than $60 \mathrm{~g} /$ 1 1,3-PDO at the 200-liter scale with crude glycerol as the carbon source, the best performance of this strain was seen with refined glycerol at the 1-liter scale: $93.7 \mathrm{~g} / \mathrm{l}$ 1,3-PDO was produced with an overall productivity of $3.3 \mathrm{~g} / \mathrm{l} / \mathrm{h}$ (Table 1). Also of note, a recent study with C. butyricum VPI 1718 demonstrated the conversion of crude glycerol to $67.9 \mathrm{~g} / \mathrm{l}(0.55 \mathrm{~g} / \mathrm{g}) 1,3-\mathrm{PDO}$ through a non-sterilized fermentation process, holding great promise for the industrial-scale conversion of glycerol to 1,3-PDO [36] (Table 1). (a)

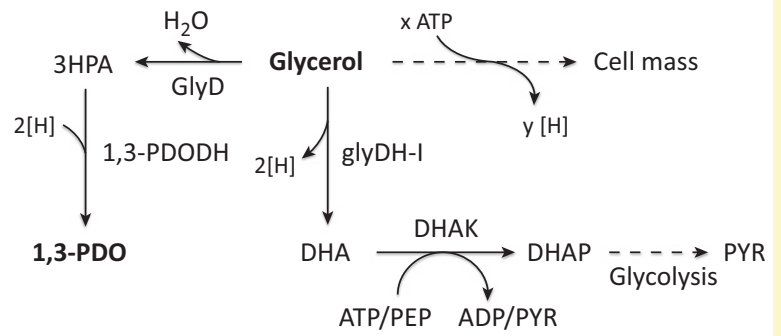

(b)

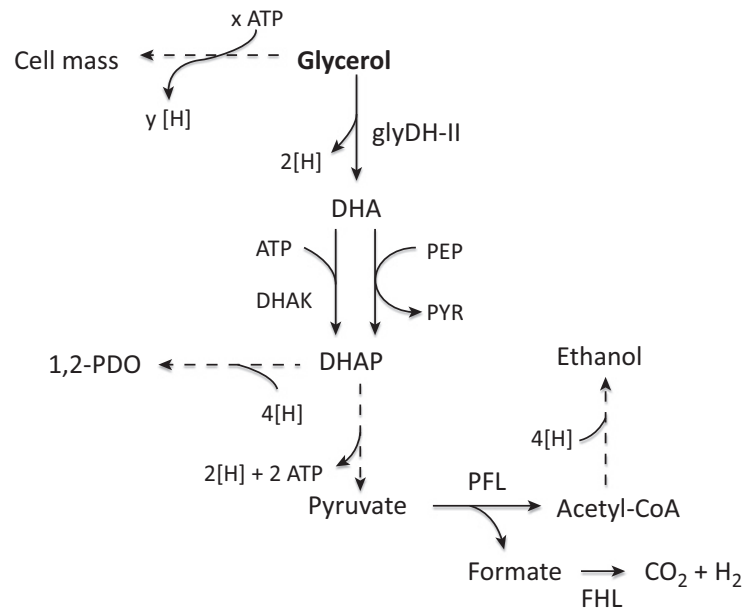

TRENDS in Biotechnology

Figure I. (a) 1,3-Propanediol (1,3-PDO)-dependent and (b) 1,2-PDO-ethanoldependent models for glycerol fermentation in microorganisms. Broken lines indicate multiple steps. Abbreviations: DHA, dihydroxyacetone; DHAK, DHA kinase; DHAP, DHA phosphate; FHL, formate hydrogen lyase; GlyD, glycerol dehydratase; glyDH-I, glycerol dehydrogenase type I; glyDH-II, glycerol dehydrogenase type II; PEP, phosphoenolpyruvate; PFL, pyruvate formatelyase; PEP, phosphoenolpyruvate; PYR, pyruvate; 1,3-PDO, 1,3-propanediol; 1,3-PDODH, 1,3-PDO dehydrogenase; 3HPA, 3-hydroxypropionaldehyde; $2[\mathrm{H}]=$ $\mathrm{NADH} / \mathrm{NADPH} / \mathrm{FADH}_{2}$.

\section{1,2-Propanediol (1,2-PDO)}

$1,2-\mathrm{PDO}$ is a commodity chemical with major roles in applications such as antifreeze and heat-transfer fluids, plasticizers and thermoset plastics, and cosmetics [42]. In contrast to 1,3-PDO, the microbial production of 1,2-PDO has focused largely on the conversion of sugars [20,43], with very few examples of the fermentative conversion of glycerol to 1.2PDO. Furthermore, studies on the conversion of sugars to 1,2-PDO have markedly lower yields than 1,3-PDO production from either sugars or glycerol [20]. This is in large part due to the fact that few microorganisms have been shown to naturally produce 1,2-PDO in large amounts, and those that have, such as Thermoanaerobacterium thermosaccharolyticum [44], lack the sufficient genetic knowledge and tools required for effective manipulation. In addition, when considering 1,2-PDO production form glycerol, it is important to note that the typical fermentative pathways for 1,2-PDO production require the conversion of the carbon source to dihydroxyacetone phosphate (DHAP) through glycolytic pathways, in contrast to the 1,3-PDO pathways that convert glycerol directly to 1,3-PDO in two-steps (Figure 4), which requires that the organism of interest possess the ability to utilize glycerol directly. 
Table 1. Selected examples of fuel and chemical production from the anaerobic fermentation of glycerol

\begin{tabular}{|c|c|c|c|c|c|}
\hline Product and organism & \begin{tabular}{|l|} 
Titer \\
(g/l)
\end{tabular} & \begin{tabular}{|l|} 
Yield \\
$(\mathrm{g} / \mathrm{g})$
\end{tabular} & $\begin{array}{l}\text { Overall productivity } \\
(\mathrm{g} / \mathrm{l} / \mathrm{h})^{\mathrm{a}}\end{array}$ & $\begin{array}{l}\text { Glycerol } \\
\text { source }\end{array}$ & Refs \\
\hline Klebsiella pneumoniae M5al & 58.8 & 0.44 & 0.92 & Refined & {$[33]^{b}$} \\
\hline Clostridium butyricum ARK102a & 93.7 & 0.52 & 3.35 & Refined $^{\mathrm{c}}$ & [41] \\
\hline Clostridium butyricum VPI 1718 & 67.9 & 0.55 & 0.78 & Crude $^{d}$ & {$[36]$} \\
\hline Clostridium acetobutylicum DG1 (pSPD5) & 84.0 & 0.54 & 1.77 & Refined $^{\mathrm{e}}$ & [38] \\
\hline \multicolumn{6}{|l|}{ Succinic acid } \\
\hline Anaerobiospirillum succiniproducens & 19.0 & 1.60 & 0.16 & Refined & [48] \\
\hline Actinobacillus succinogenes & 29.3 & 0.80 & - & Refined & [49] \\
\hline Escherichia coli & 12.0 & 1.02 & 0.08 & Refined & {$[50]$} \\
\hline \multicolumn{6}{|l|}{ Propionic acid } \\
\hline Klebsiella oxytoca FMCC-197 & $25.2^{\mathrm{g}}$ & 0.20 & 0.29 & Crude & {$[66]$} \\
\hline Escherichia coli & 19.8 & 0.47 & 0.20 & Crude & [68] \\
\hline \multicolumn{6}{|l|}{ n-Butanol } \\
\hline Clostridium pasteurianum MBEL_GLY2 & 17.8 & 0.30 & 0.30 & Refined & {$[76]$} \\
\hline Clostridium pasteurianum DSM 525 & 7.2 & $0.31^{\mathrm{h}}$ & 0.15 & Thin stillage & {$[77]$} \\
\hline
\end{tabular}

${ }^{a}$ Overall productivity calculated by dividing product titer by total fermentation time reported.

${ }^{\mathrm{b}}$ Results obtained in 5000-liter pilot scale fermentor.

${ }^{\mathrm{c}}$ Reported values obtained with refined glycerol. Use of crude glycerol resulted in titers of $61.5 \mathrm{~g} / \mathrm{l}$ at 200 -liter scale.

${ }^{\mathrm{d}}$ Fermentation conducted under non-sterile conditions.

${ }^{e}$ Reported values obtained with refined glycerol. No significant differences in 1,3-PDO productivity with the use of crude glycerol when lower feed glycerol concentrations used.

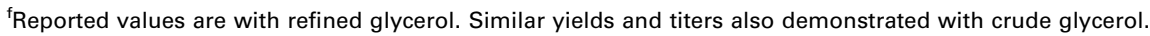

${ }^{g}$ Ethanol co-produced along with $50.1 \mathrm{~g} / \mathrm{l}$ of 1,3-PDO.

hYield calculated from total amount of glycerol and lactate consumed from thin stillage.

The recent discovery that $E$. coli is able to utilize glycerol under fermentative conditions via an ethanol-1,2PDO-dependent model [19] opened the possibility to engineer $E$. coli for the conversion of glycerol to 1,2-PDO [45].

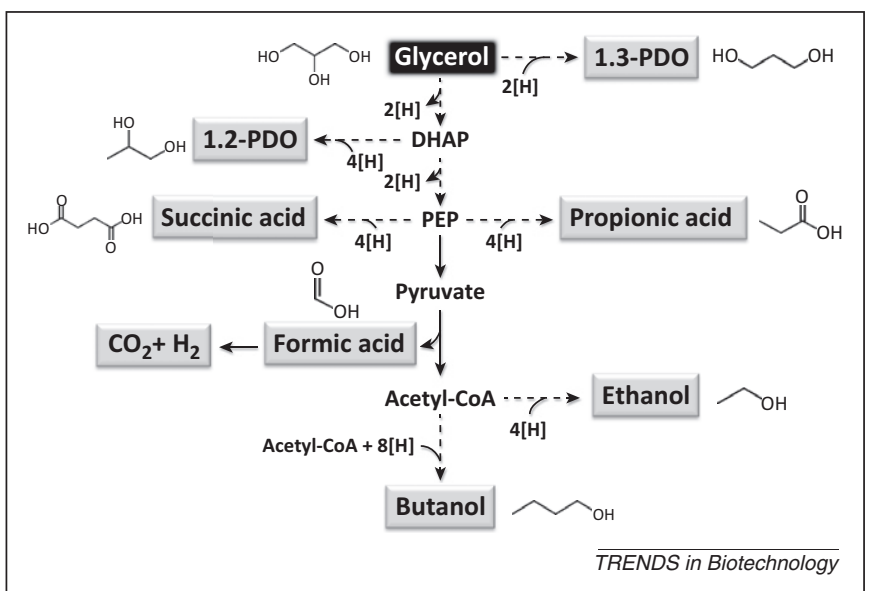

Figure 4. Anaerobic fermentation of glycerol for the production of fuels and reduced chemicals. Products in gray shaded boxes represent those whose synthesis has been demonstrated from the anaerobic fermentation of glycerol. The high degree of reduced carbon atoms in glycerol results in pathways to each product representing a redox-balanced or redox-consuming conversion. In each case, the theoretical maximum yield is higher than that obtained from common sugars, such as glucose or xylose. Broken lines represent multiple reaction steps. Abbreviations: DHAP, dihydroxyacetone-phosphate; PEP, phosphoenolpyruvate.
Here, 1,2-PDO production from glycerol by $E$. coli, which has been shown to produce 1,2-PDO only in very small amounts during fermentative glycerol metabolism, was increased by engineering a functional 1,2-PDO pathway by (i) combination of overexpression of genes involved in its synthesis from the key intermediate DHAP and (ii) manipulation of the fermentative glycerol utilization pathway. After deletion of the fermentative pathways for acetate and lactate production, representing competing byproducts, the final engineered $E$. coli produced $5.6 \mathrm{~g} / \mathrm{l}$ $1,2-\mathrm{PDO}$, at a yield of $0.21 \mathrm{~g} 1,2-\mathrm{PDO} / \mathrm{g}$ glycerol consumed [45] (Table 1).

\section{Succinic acid}

Currently used in the agricultural, food, and pharmaceutical industries, succinic acid can easily be converted into a wide variety of products including biodegradable plastics and green solvents $[46,47]$. Although there have been numerous studies on the conversion of sugars to succinic acid [47], only a small number of reports document the anaerobic conversion of glycerol to succinic acid. Succinic acid production represents a redox balanced pathway from glycerol [9], which should facilitate the ease at which high yield and titers of succinic acid can be produced utilizing glycerol as a carbon source. In a study with Anaerobiospirillum succiniproducens, succinic acid production from glycerol or glucose fermentations with a carbon dioxide 
atmosphere, revealed that glycerol fermentation produced significantly less acetic acid. Utilizing a fed-batch culture in which both glycerol and yeast extract were fed, $19.0 \mathrm{~g} / \mathrm{l}$ of succinic acid at a yield of $1.60 \mathrm{~g}$ succinic acid/g glycerol consumed was produced by $A$. succiniproducens with minimal byproduct formation [48] (Table 1). Actinobacillus succinogenes has also been exploited for the conversion of glycerol to succinic acid utilizing anaerobic fermentations with carbon dioxide sparging [49]. The authors performed a directed evolution procedure to increase the tolerance of $A$. succinogenes to glycerol, and the final strain obtained with this procedure was able to produce $29.3 \mathrm{~g} / \mathrm{l}$ succinic acid at a yield of $0.80 \mathrm{~g}$ succinic acid/g glycerol consumed (Table 1).

The fermentative conversion of glycerol to succinic acid has also been reported by metabolically engineered $E$. coli [50]. Here, a chromosomal promoter mutation was used to upregulate $p c k$, encoding the gluconeogenic enzyme PEP carboxykinase, which generates an ATP molecule during PEP carboxylation. This provides the engineered strain with a route to succinic acid that generates ATP, as the traditional pathway for PEP carboxylation through PEP carboxylase (encoded by ppc) does not generate this additional ATP. Combining this mutation with the inactivation of pyruvate formate lyase (encoded by $p f B$ ), minimizes byproduct formation. Disruption of the fermentative glycerol dissimilation pathway through a ptsI deletion resulted in a strain producing $12 \mathrm{~g} / \mathrm{l}$ succinic acid at a yield $80 \%$ of the theoretical maximum (1.0 on a molar basis) from glycerol under anaerobic conditions [50] (Table 1).

\section{Propionic acid}

The production of propionic acid, a chemical widely used in cellulose plastics, herbicides, and perfumes, has been studied with, Gram-positive, non-spore forming anaerobes of the Propionibacterium genera, which have been shown to possess the ability to utilize glycerol under fermentative conditions [51-53]. Propionibacterium acidipropionici produces more propionic acid with glycerol than with glucose [51]. This strain was capable of producing propionic acid titers of $42 \mathrm{~g} / \mathrm{l}$ from $80 \mathrm{~g} / \mathrm{l}$ initial glycerol with a maximum productivity of $0.36 \mathrm{~g} / \mathrm{l} / \mathrm{h}$ [51] (Table 1). Moreover, acetic acid (the main byproduct in fermentation with glucose) production was decreased with the use of glycerol as the carbon source.

\section{Ethanol and co-products}

Ethanol has emerged as an important renewable and sustainable fuel that currently represents the most widely produced biofuel in the USA [54]. Industrial production of ethanol is dominated by the conversion of sugars from starch or sucrose to ethanol by the yeast Saccharomyces cerevisiae [55]. Significant research efforts have focused on engineering other microorganisms to produce maximal ethanol yields and titers from lignocellulosic derived sugars [56-60]. Despite the success of these engineering efforts, the oxidation state of sugars such as glucose dictates that about half of their weight is lost as $\mathrm{CO}_{2}$ during the fermentation process (Figure 3 ), reducing the product yield. By contrast, the reduced nature of glycerol permits the co-production of ethanol and formic acid (or ethanol and hydrogen), enabling increased overall product yields compared to sugars. Furthermore, the recent discoveries that E. coli [19] and Paenibacillus macerans [61] ferment glycerol in an ethanol-1,2-PDO-dependent manner (Box 2) opens the possibly to use these organisms for the direct conversion of glycerol into ethanol. In addition to these strains, Klebsiella planticola has been shown to co-produce ethanol and formic acid during the fermentative utilization of glycerol [62], and Enterobacter aerogenes has been utilized for the anaerobic conversion of glycerol to ethanol and hydrogen [63,64] (Table 1). Glycerol has also been converted to ethanol and small amounts of formic and lactic acid, by a newly isolated strain of $C$. freundii (designated as FMCC-207) [65]. This strain was capable of producing $14.5 \mathrm{~g} / \mathrm{l}$ ethanol at a yield of $0.45 \mathrm{~g} / \mathrm{g}$ with a volumetric productivity of $\sim 0.7 \mathrm{~g} / \mathrm{l} / \mathrm{h}$ (Table 1 ). A newly isolated Klebsiella oxytoca strain (FMCC-197) showed the ability to coproduce high titers of ethanol $(25.2 \mathrm{~g} / \mathrm{l})$ along with 1,3-PDO $(50.1 \mathrm{~g} / \mathrm{l})$ and lactic acid $(16.8 \mathrm{~g} / \mathrm{l})$ during fed-batch fermentations with crude glycerol [66] (Table 1).

Despite the high ethanol yields from glycerol fermentation in $E$. coli, the main drawback from an industrial standpoint is the low rate of glycerol consumption, leading to unfavorable ethanol productivities compared to those obtained from the fermentation of sugars [67]. Glycolytic flux during glycerol fermentation with $E$. coli is almost exclusively controlled by the enzymes glycerol dehydrogenase (encoded by gldA) and dihydroxyacetone kinase (encoded by $d h a K L M$ ) [68]. Overexpression of these two enzymes led to downstream production of nearly $20 \mathrm{~g} / \mathrm{l}$ ethanol from crude glycerol, significantly improving the productivity of this process (Table 1). It is also important to note that the reduced nature of carbon atoms in glycerol dictated that minimal byproducts were formed (ethanol produced at $92 \%$ of the theoretical maximum of $1 \mathrm{~mol} / \mathrm{mol}$ ) and hydrogen was co-produced in equimolar ratios to ethanol [68], the latter not possible with the use of sugars.

$E$. coli has also been engineered to improve hydrogen and ethanol co-production through adaptive evolution and chemical mutagenesis, combined with a selection method based on increased growth on glycerol [69]. Here, an E. coli strain in which the frdC gene was deleted to reduce the repression of hydrogen synthesis during glycerol fermentation and avoid re-utilization of hydrogen underwent chemical mutagenesis. The final isolated mutant showed a 20 -fold increase in hydrogen production $(0.68 \mathrm{mmol} / \mathrm{l} / \mathrm{h})$, accompanied by a fivefold increase in ethanol production and growth rate [69].

\section{n-Butanol}

The high energy content and lower hygroscopicity of nbutanol have made it an attractive alternative to ethanol [54]. Historically, n-butanol has been produced biologically from sugars through the acetone-butanol-ethanol (ABE) fermentation by $C$. acetobutylicum and related organisms [70]. Recently E. coli strains have been engineered to produce high titers and yields of n-butanol from glucose [71-73], which holds great promise for the conversion of lignocellulosic-derived feedstocks to n-butanol [74]. At present, the natural ability for certain organisms possessing $\mathrm{ABE}$ fermentation characteristics to ferment glycerol, 
such as C. pasteurianum [26], have provided the only viable route for the production of n-butanol from glycerol. Although $C$. pasteurianum has been shown to produce significant amounts of n-butanol during glycerol fermentation [75], low rates of glycerol utilization and productivities of product synthesis would likely limit the economic viability of such a process on the industrial scale. In an effort to alleviate some of these potential issues, a hyper butanol-producing mutant of $C$. pasteurianum has been developed using chemical mutagenesis and selection [76]. After chemical mutagenesis and screening of mutants based on their ability to produce high amounts of n-butanol, process optimization with the hyper butanol producing mutant of C. pasteurianum enabled the production of $17.8 \mathrm{~g} / \mathrm{l} \mathrm{n}$-butanol $(27.0 \mathrm{~g} / \mathrm{l}$ total solvents produced) from glycerol at a yield of $0.30 \mathrm{~g} \mathrm{n}$-butanol/g glycerol consumed with a maximum productivity of $0.43 \mathrm{~g} / \mathrm{l} / \mathrm{h}$ (Table 1 ).

C. pasteurianum DSM 525 is also able to produce significant amounts of butanol from thin stillage produced as a direct byproduct of an ethanol fermentation process [77]. Starting with thin stillage containing around $20 \mathrm{~g} / \mathrm{l}$ glycerol along with significant amounts of lactic acid, acetic acid, and 2,3-butanediol, this strain was capable of producing $7.2 \mathrm{~g} / \mathrm{l}$ n-butanol in 48 hours with near complete consumption of the glycerol and lactic acid initially present [77]. Although not a pure glycerol fermentation process due to the presence of additional carbon sources, the ability of this organism to directly utilize this feedstock is a significant finding. Thin stillage currently represents the largest source of waste glycerol that can be converted to value added products (along with the other carbon sources present).

\section{Conclusions and future perspectives}

Availability, low price, and high degree of reduction make glycerol an attractive carbon source for the production of fuels and reduced chemicals. Glycerol's reduced state enables synthesis of reduced products at higher yields compared to common sugars. Although the number of organisms able to use this reduced carbon source under fermentative conditions is limited, $K$. pneumoniae, $C$. pasteurianum, and C. butyricum have been exploited to produce several industrially relevant compounds at high yields and titers including 1,3-PDO and n-butanol. In addition to these products, microorganisms naturally producing compounds such as propionic acid and succinic acid have also been utilized to produce these products through the anaerobic fermentation of glycerol.

Although some organisms can convert glycerol into valuable products, their use on the industrial scale may prove difficult. Genetic tools and physiological knowledge of these organisms are lacking, making their genetic manipulation for improved production of native and nonnative products challenging. $E$. coli can ferment glycerol in the absence of external electron acceptors, thus providing an alternative platform for the conversion of glycerol into a wide array of fuels and chemicals. Currently, E. coli has been engineered to produce a number of target products during the anaerobic fermentation of glycerol, including 1,2-PDO, ethanol, hydrogen, and succinic acid. Although the current array of compounds that can be produced with glycerol fermentation is limited compared to those produced from sugars, continued interest and advancements in the fields of metabolic engineering and synthetic biology will further the portfolio of available compounds and serve to fully exploit the advantageous nature of glycerol for the production of fuels and reduced chemicals.

\section{Acknowledgments}

This work was supported by grants from the US National Science Foundation (CBET-0645188 and BES-0331388/BES-0601549) and the National Research Initiative of the US Department of Agriculture Cooperative State Research, Education, and Extension Service (200535504-16698).

\section{References}

1 Hirsch, R.L. et al. (2006) Peaking of world oil production and its mitigation. AICHE J. 52, 2-8

2 Kerr, R.A. (2007) Global warming is changing the world. Science 316, 188-190

3 Clomburg, J.M. and Gonzalez, R. (2010) Biofuel production in Escherichia coli: the role of metabolic engineering and synthetic biology. Appl. Microbiol. Biotechnol. 86, 419-434

4 Dellomonaco, C. et al. (2010) The path to next generation biofuels: successes and challenges in the era of synthetic biology. Microb. Cell Fact. 9, http://dx.doi.org/10.1186/1475-2859-9-3

5 Fischer, C.R. et al. (2008) Selection and optimization of microbial hosts for biofuels production. Metab. Eng. 10, 295-3046

6 Peralta-Yahya, P.P. et al. (2012) Microbial engineering for the production of advanced biofuels. Nature 488,320-328

7 Rude, M.A. and Schirmer, A. (2009) New microbial fuels: a biotech perspective. Curr. Opin. Microbiol. 12, 274-281

8 Connor, M.R. and Liao, J.C. (2009) Microbial production of advanced transportation fuels in non-natural hosts. Curr. Opin. Biotechnol. 20 , 307-315

9 Yazdani, S.S. and Gonzalez, R. (2007) Anaerobic fermentation of glycerol: a path to economic viability for the biofuels industry. Curr. Opin. Biotechnol. 18, 213-219

$10 \mathrm{Kamm}$, B. and Kamm, M. (2007) Biorefineries - multi product processes. In White Biotechnology (Ulber, R. and Sell, D., eds), pp. 175-204, Springer-Verlag

11 Khanal, S.K. et al. (2008) Bioenergy and biofuel production from wastes/residues of emerging biofuel industries. Water Environ. Res. $80,1625-1647$

12 Rausch, K.D. and Belyea, R.L. (2006) The future of coproducts from corn processing. Appl. Biochem. Biotechnol. 128, 47-86

13 Zeng, A-P. and Biebl, H. (2002) Bulk chemicals from biotechnology: the case of 1,3-propanediol production and the new trends. Adv. Biochem. Eng. Biotechnol. 74, 239-259

14 Claude, S. et al. (2000) Commercialisation of glycerol. In Proceedings of Chemical-Technical Utilisation of Vegetable Oils Conference (Stelter, W. et al., eds), pp. 129-146, Gutzow: FNR

15 Oren, A. (2005) A hundred years of Dunaliella research: 1905-2005. Saline Syst. 1, 2

16 Nielsen, J. et al. (2003) Bioreaction Engineering Principles, Kluwer Adademic/Plenum Publishers

17 Neidhardt, F.C. et al. (1996) Escherichia coli and Salmonella: Cellular and Molecular Biology, (2nd edn), ASM Press

18 Murarka, A. et al. (2008) Fermentative utilization of glycerol by Escherichia coli and its implications for the production of fuels and chemicals. Appl. Environ. Microbiol. 74, 1124-1135

19 Gonzalez, R. et al. (2008) A new model for the anaerobic fermentation of glycerol in enteric bacteria: trunk and auxiliary pathways in Escherichia coli. Metab. Eng. 10, 234-245

20 Zeng, A.P. and Sabra, W. (2011) Microbial production of diols as platform chemicals: recent progresses. Curr. Opin. Biotechnol. 22, 749-757

21 Schutz, H. and Radler, F. (1984) Anaerobic reduction of glycerol to 1,3propanediol by Lactobaciluus brevis and Lactobacillus buchneri. Syst. Appl. Microbiol. 5, 169-178

22 Sobolov, M. and Smiley, K.L. (1960) Metabolism of glycerol by an acrolein-forming Lactobacillus. J. Bacteriol. 79, 261-266 
23 Humphreys, F.B. (1924) Formation of acrolein from glycerol by $B$. welchii. J. Infect. Dis. 35, 282-290

24 Homann, T. et al. (1990) Fermentation of glycerol to 1,3-propanediol by Klebsiella and Citrobacter strains. Appl. Microbiol. Biotechnol. 33, 121126

25 Streekstra, H. et al. (1987) Overflow metabolism during anaerobic growth of Klebsiella aerogenes NCTC 418 on glycerol and dihydroxyacetone in chemostat culture. Arch. Microbiol. 147, 268275

26 Dabrock, B. et al. (1992) Parameters afftecting solvent prodcution by Clostridium pasteurianum. Appl. Environ. Microbiol. 58, 1233-1239

27 Saint-Amans, S. et al. (2001) Regulation of carbon and electron flow in Clostridium butyricum VPI 3266 grown on glucose-glycerol mixtures. J. Bacteriol. 183, 1748-1754

28 Heyndrickx, M. et al. (1991) The fermentation of glycerol by Clostridium butyricum LMG-1212T2 and LMG-1213T1 and C. pasteurianum LMG-3285. Appl. Microbiol. Biotechnol. 34, 637642

29 Biebl, H. et al. (1992) Glycerol conversion to 1,3-propanediol by newly isolated Clostridia. Appl. Microbiol. Biotechnol. 36, 592-597

30 Biebl, H. (2001) Fermentation of glycerol by Clostridium pasteurianum: batch and continuous culture studies. J. Ind. Microbiol. Biotechnol. 27, 18-26

31 Barbirato, F. et al. (1998) 1,3-propanediol production by fermentation: an interesting way to valorize glycerin from the ester and ethanol industries. Ind. Crops Prod. 7, 281-289

$32 \mathrm{Mu}, \mathrm{Y}$. et al. (2006) Microbial production of 1,3-propanediol by Klebsiella pneumoniae using crude glycerol from biodiesel preparations. Biotechnol. Lett. 28, 1755-1759

33 Cheng, K.K. et al. (2007) Pilot-scale production of 1,3-propanediol using Klebsiella pneumoniae. Process Biochem. 42, 740-744

34 Gonzalez-Pajuelo, M. et al. (2006) Microbial conversion of glycerol to 1,3-propanediol: physiological comparison of a natural producer, Clostridium butyricum VPI 3266, and an engineered strain, Clostridium acetobutylicum DG1(pSPD5). Appl. Environ. Microbiol. 72, 96-101

35 Papanikolaou, S. et al. (2000) High production of 1,3-propanediol from industrial glycerol by a newly isolated Clostridium butyricum strain. $J$. Biotechnol. 77, 191-208

36 Chatzifragkou, A. et al. (2011) Production of 1,3-propanediol by Clostridum butyricum growing on biodiesel-derived crude glycerol through a non-sterilized fermentation process. Appl. Microbiol. Biotechnol. 91, 101-112

37 Saintamans, S. et al. (1994) High production of 1,3-propanediol from glycerol by Clostridium butyricum VPI-3266 in a simply controlled fedbatch system. Biotechnol. Lett. 16, 831-836

38 Gonzalez-Pajuelo, M. et al. (2005) Metabolic engineering of Clostridium acetobutylicum for the industrial production of 1,3-propanediol from glycerol. Metab. Eng. 7, 329-336

39 Willke, T. and Vorlop, K. (2008) Biotransformation of glycerol into 1,3propanediol. Eur. J. Lipid Sci. Technol. 110, 831-840

40 Ringel, A.K. et al. (2012) An improved screening method for microorganisms able to convert crude glycerol to 1,3-propanediol and to tolerate high product concentrations. Appl. Microbiol. Biotechnol. 93, 1049-1056

41 Wilkens, E. et al. (2012) High-level production of 1,3-propanediol from crude glycerol by Clostridium butyricum AKR102a. Appl. Microbiol. Biotechnol. 93, 1057-1063

42 Shelley, S. (2007) A renewable route to propylene glycol. Chem. Eng. Prog. 103, 6-9

43 Bennett, G.N. and San, K.Y. (2001) Microbial formation, biotechnological production and applications of 1,2-propanediol. Appl. Microbiol. Biotechnol. 55, 1-9

44 Sanchezriera, F. et al. (1987) Influence of envrionmental factors in the production of $\mathrm{R}(-)-1,2$-propanediol by Clostridium thermosaccharolyticum. Biotechnol. Lett. 9, 449-454

45 Clomburg, J.M. and Gonzalez, R. (2011) Metabolic engineering of Escherichia coli for the production of 1,2-propanediol from glycerol. Biotechnol. Bioeng. 108, 867-879

46 McKinlay, J.B. et al. (2007) Prospects for a bio-based succinate industry. Appl. Microbiol. Biotechnol. 76, 727-740

47 Song, H. and Lee, S.Y. (2006) Production of succinic acid by bacterial fermentation. Enzyme Microb. Technol. 39, 352-361
48 Lee, P.C. et al. (2001) Succinic acid production with reduced byproduct formation in the fermentation of Anaerobiospirillum succiniciproducens using glycerol as a carbon source. Biotechnol. Bioeng. 72, 41-48

49 Vlysidis, A. et al. (2011) Glycerol utilisation for the production of chemicals: conversion to succinic acid, a combined experimental and computational study. Biochem. Eng. J. 58-59, 1-11

50 Zhang, X.L. et al. (2010) Fermentation of glycerol to succinate by metabolically engineered strains of Escherichia coli. Appl. Environ. Microbiol. 76, 2397-2401

51 Barbirato, F. et al. (1997) Propionic acid fermentation from glycerol: comparison with conventional substrates. Appl. Microbiol. Biotechnol. 47, 441-446

52 Himmi, E.H. et al. (2000) Propionic acid fermentation of glycerol and glucose by Propionibacterium acidipropionici and Propionibacterium freudenreichii ssp shermanii. Appl. Microbiol. Biotechnol. 53, 435-440

53 Bories, A. et al. (2004) Glycerol fermentation with Propionibacteria and optimisation of the production of propionic acid. Sci. Aliments 24, 121135

54 Fortman, J.L. et al. (2008) Biofuel alternatives to ethanol: pumping the microbial well. Trends Biotechnol. 26, 375-381

55 Gray, K.A. et al. (2006) Bioethanol. Curr. Opin. Chem. Biol. 10, 141146

56 Ingram, L.O. et al. (1999) Enteric bacterial catalysts for fuel ethanol production. Biotechnol. Prog. 15, 855-866

$57 \mathrm{Kim}$, Y.et al. (2007) Construction of an Escherichia coli K-12 mutant for homoethanologenic fermentation of glucose or xylose without foreign genes. Appl. Environ. Microbiol. 73, 1766-1771

58 Trinh, C.T. et al. (2008) Minimal Escherichia coli cell for the most efficient production of ethanol from hexoses and pentoses. Appl. Environ. Microbiol. 74, 3634-3643

59 Yomano, L.P. et al. (2008) Re-engineering Escherichia coli for ethanol production. Biotechnol. Lett. 30, 2097-2103

60 Zhang, M. et al. (1995) Metabolic engineering of a pentose metabolism pathway in ethanologenic Zymomonas mobilis. Science 267, 240-243

61 Gupta, A. et al. (2009) Anaerobic fermentation of glycerol in Paenibacillus macerans: metabolic pathways and environmental determinants. Appl. Environ. Microbiol. 75, 5871-5883

62 Jarvis, G.N. et al. (1997) Formate and ethanol are the major products of glycerol fermentation produced by a Klebsiella planticola strain isolated from red deer. J. Appl. Microbiol. 83, 166-174

63 Ito, T. et al. (2005) Hydrogen and ethanol production from glycerolcontaining wastes discharged after biodiesel manufacturing process. $J$. Biosci. Bioeng. 100, 260-265

64 Markov, S.A. et al. (2011) Bioreactor for glycerol conversion into $\mathrm{H}_{2}$ by bacterium Enterobacter aerogenes. Int. J. Hydrogen Energy 36, 262266

65 Metsoviti, M. et al. (2012) Screening of bacterial strains capable of converting biodiesel-derived raw glycerol into 1,3-propanediol, 2,3butanediol and ethanol. Eng. Life Sci. 12, 57-68

66 Metsoviti, M. et al. (2012) Production of 1,3-propanediol, 2,3-butanediol and ethanol by a newly isolated Klebsiella oxytoca strain growing on biodiesel-derived glycerol based media. Process Biochem. http:// dx.doi.org/10.1016/j.procbio.2012.06.011

67 Dharmadi, Y. et al. (2006) Anaerobic fermentation of glycerol by Escherichia coli: a new platform for metabolic engineering. Biotechnol. Bioeng. 94, 821-829

68 Cintolesi, A. et al. (2012) Quantitative analysis of the fermentative metabolism of glycerol in Escherichia coli. Biotechnol. Bioeng. 109, 187-198

69 Hu, H.B. and Wood, T.K. (2010) An evolved Escherichia coli strain for producing hydrogen and ethanol from glycerol. Biochem. Biophys. Res. Commun. 391, 1033-1038

70 Maddox, I.S. (1989) The acetone-butanol-ethanol fermentation: recent progress in technology. Biotechnol. Genet. Eng. Rev. 7, 189-220

71 Dellomonaco, C. et al. (2011) Engineered reversal of the $\beta$-oxidation cycle for the synthesis of fuels and chemicals. Nature 476, 355359

72 Shen, C.R. et al. (2011) Driving forces enable high-titer anaerobic 1butanol synthesis in Escherichia coli. Appl. Environ. Microbiol. 77, 2905-2915

73 Bond-Watts, B.B. et al. (2011) Enzyme mechanism as a kinetic control element for designing synthetic biofuel pathways. Nat. Chem. Biol. 7, $222-227$ 
74 Bokinsky, G. et al. (2011) Synthesis of three advanced biofuels from ionic liquid-pretreated switchgrass using engineered Escherichia coli. Proc. Natl. Acad. Sci. U.S.A. 108, 19949-19954

75 Taconi, K.A. et al. (2009) Growth and solvent production by Clostridium pasteurianum ATCC 6013 utilizing biodiesel-derived crude glycerol as the sole carbon source. Environ. Prog. Sustainable Energy 28, 100-110

76 Malaviya, A. et al. (2012) Continuous butanol production with reduced byproducts formation from glycerol by a hyper producing mutant of Clostridium pasteurianum. Appl. Microbiol. Biotechnol. 93, 1485-1494
77 Ahn, J.H. et al. (2011) Butanol production from thin stillage using Clostridium pasteurianum. Bioresour. Technol. 102, 4934-4937

78 Bouvet, O.M.M. et al. (1995) Taxonomic diversity of anaerobic glycerol dissimilation in the Enterobacteriaceae. Res. Microbiol. 146, 279-290

79 Both, I.R. (2005) Glycerol and methylglyoxal metabolism. In EcoSalEscherichia coli and Salmonella: Cellular and Molecular Biology (Curtis, R., III et al. eds.) ASM Press (http://www.ecosal.org)

80 Sun, J. et al. (2003) Comparative genomic analysis of dha regulon and related genes for anaerobic glycerol metabolism in bacteria. Biotechnol. Prog. 19, 263-272 